\title{
NEVRALGIA VAGOGLOSSOFARÍNGEA
}

\author{
S. L. ROSSITTI * - R.J. BALBO ** - A. SPERLESCU ***
}

RESUMO - Revisão dos conceitos vigentes sobre a clínica, patogenia, diagnóstico diferencial e tratamento da neuralgia vagoglossofaríngea, e relato de três casos operados por neurotomia intracraniana do n. IX e n. $X$ (parcial). A anatomia da inervação sensitiva das regiões profundas da face e transição cervicofacial, com suas implicações clínicas, e a teoria da compressão neurovascular na patogenia das disfunções ditas «hiperativas» dos nervos cranianos, são comentadas criticamente.

\begin{abstract}
Vagoglossopharyngeal neuralgia.
SUMMARY - Review of current opinions concerning clinical presentation, etiology, differential diagnosis and management of vagoglossopharyngeal neuralgia. Three cases are reported, treated by intracranial section of $n$. IX (alone in one case) and upper rootlets of n. X. In two patients no vascular compression of the nerves was observed. In one case an atheromatous elongated basilar artery was observed compressing and deforming the medulla oblonga at the entry zone of nerves IX-X. In this case, pain paroxysms recurred, some weeks after surgery, and the patient underwent re-operation (microvascular decompression of the medulla) with good outcome. Clinical implications of the complex sensitive innervation of profound regions of the face and cervicofacial region are emphasized. Certain circumstances, such as gustatory pain due to sympathetic denervation of parotid gland, the necktongue syndrome and oropharyngeal pain by irritation of the first cervical spinal nerve (during lateral suboccipital puncture), point to the fact that in this region similar symptoms may be provoked by mechanisms involving different peripheric pain pathways. The theory of microvascular compression in the pathogeny of cranial nerve «hyperactive» dysfunctions is critically commented.
\end{abstract}

O tic douloureux ou neuralgia vagoglossofaríngea assemelha-se em muitos aspectos à neuralgia trigeminal, sendo todavia mais rara. Sua frequência é estimada em 1 para 70 a 100 casos de neuralgia trigeminal. Os termos «algia velofaríngea» e «neuralgia glossofaríngea»* são inadequados, pois a dor não se limita ao véu palatino e faringe e é nítido o envolvimento de fibras vagais, razão pela qual White e Sweet 18 cunharam o termo «neuralgia vagoglossofaríngea». A esses autores devemos uma das mais minuciosas descrições dessa doença, que pode assumir formas clínicas e severidade tão variáveis a ponto de casos «atípicos» serem antes a regra que a exceção. O diagnóstico baseia-se na história clínica e na observação de ataques. Tem igual incidência entre homens e mulheres, início entre 30 e 50 anos, acomete mais freqüentemente o lado esquerdo e é bilateral em menos de $2 \%$ dos casos. Os ataques de dor na maioria dos casos têm início em um lado da garganta ou região tonsilar, irradiando-se pela tuba auditiva, membrana timpânica, canal auditivo externo,

Departamento de Neuro-Psiquiatria da Faculdade de Ciências Médicas da Pontifícia Universidade Católica de Campinas (PUCCAMP) e Departamento de Neurocirurgia do Hospital Vera Cruz (HVC), Campinas: * Médico Residente; ** Professor Adjunto e Diretor do Departamento de Neurocirurgia do HVC; *** Professor Assistente e Neurocirurgião do HVC.

Dr. Sandro L. Rossitti - Departamento de Neurocirurgia, Hospital Vera Cruz - Av. Andrade Neves 402 - 13020 Campinas SP - Brasil. 
porções do pavilhão auditivo e áreas cutâneas adjacentes, ângulo da mandíbula, terço posterior da língua, naso- oro- ou laringo-faringe, epiglote e laringe. A dor pode iniciar-se em qualquer dessas regiões e irradiar-se ou não para qualquer outra. Ela é mais freqüentemente irradiada que restrita e sua localização pode variar mesmo em paroxismos de um mesmo paciente. Quando a dor se restringe ao ouvido e tuba auditiva, pode ser chamada neuralgia de Jacobson e, quando restrita à laringe, neuralgia laríngea ou de Aveílis. As funções sensitiva e motora das áreas afetadas são normais. Os ataques podem ser espontâneos, mas, em geral, são desencadeados em zonas de gatilho na faringe, fauce, tonsila ou base da língua, pelo ato de deglutir (o desencadeante mais freqüente), mastigar, falar, tossir, espirrar, lavar o ouvido, e até a rotação ou inclinação da cabeça, entre outros. Alguns pacientes, para evitar a dor evitam deglutir a saliva, a qual escorre constantemente pelos cantos da boca o acúmulo ue saliva sobre a zona de gatilho durante o sono pode causar dor e despertar o paciente; a inclinação da cabeça para o lado oposto ao da dor durante a deglutição diminui o contato do alimento com a zona de gatilho, segundo alguns pacientes; a dificuldade para se alimentar pode levar a caquexia. Aqueles com dor piecipitada pela fonação por vezes são forçados a se comunicar pela escrita; verbalizações específicas podem causar dor, como as consoantes guturais da língua inglesa $\left\langle\mathrm{g} »,\left\langle\mathrm{k} » \mathrm{e}\left\langle\mathrm{h} »^{\mathrm{T}} \mathrm{o}\right.\right.\right.$. A anestesia local das zonas de gatilho suprime os paroxismos dolorosos, procedimento esse ao mesmo tempo diagnóstico e paliativo. Estímulos tácteis, térmicos e gustatórios, bem como a massagem do seio carotídeo, também desencadeiam ataques. A dor ocorre em salvas de dias ou meses, seguidos por períodos de remissão, pode durar de alguns segundos a minutos, tem início súbito ou insidioso, caráter agudo e excruciante ou lento, como queimação e, durante os surtos, ocorre esporadicamente ou é tão freqüente que se torna incapacitante. Sintomas associados a dor são descritosio.i8 sensações não-dolorosas (parestesias na língua e faringe, dificuldade para ouvir sons graves e sussurros, vertigem e tinnitus), atos motores involuntários (tosse, vômito, fonação, soluço, que podem iniciar novos ataques), fenômenos autonômicos (hipersecreção parotídea, sudorese, lacrimação, midríase e hiperemia da face, homolaterais a dor) e fenômenos de irritação do seio carotídeo e n. de Hering (bradicardia, assístolia, síncope e convulsões generalizadas, por vezes impondo-se o diagnóstico diferencial com as epilepsias) aos quais associa-se a inibição da atividade simpática periférica.

Com relação à etiologia, foram observadas infecção $\operatorname{local}^{10}{ }^{18}{ }^{18}$ (abscesso peritonsilar, otite média bacteriana, tuberculose da aritenóide, abscessos profundos da região mandibular) e trauma ${ }^{18}$ (por projétil de arma de fogo e tonsilectomías unusualmente traumáticas) precedendo o início de paroxismos típicos. O processo estilóide, quando muito alongado (seu comprimento no adulto varia de 2,5 a $3 \mathrm{~cm}$; raramente até $7,5 \mathrm{~cm}$ ), pode irritar a parede lateral da faringe durante a deglutição, no momento em que ela é deslocada superior e lateralmente pela ação do músculo (m.) estilofaríngeo io.is. A ressecção do processo estilóide alongado teve bons resultados em 11 dç 12 casos coletados na literatura por White e $\mathrm{Sweet}^{18}$. A possibilidade de uma lesão central, ao nível do tracto espinhal do nervo (n.) V (que também recebe fibras do n. VII, n. IX, n. X e nervos espinhais superiores) já foi aventada; a topografia das fibras da terceira divisão (V3) do $\mathrm{n}$. V, situadas adjacentes às do $\mathrm{n}$. $\mathrm{X}$ sugere essa hipótese, dado que a neuralgia combinada trigeminal e vagoglossofaríngea sempre acomete V 3 ( é bem estabelecida uma somatotopia, na seqüência de dorsomedial para lateral: VII, IX, X, V3, V2, VI). Depõe contra essa hipótese o fato de não se ter observado essa neuralgia combinada associada a esclerose múltipla ou a outras lesões bem definidas desse tracto, como nos 5 pacientes relatados por Mracek" ${ }^{\prime \prime 4}$, com neuralgia trigeminal, durante o tratamento cirúrgico (tractotorr.ia de Sjõqvist) encontrou-se nesses pacientes uma artéria (a.) cerebelar posterior inferior alongada e tortuosa, pressionando e distorcendo o bulbo sobre o tracto espinhal no n. V; o autor opera com anestesia local e observou que o toque ou tração da artéria provocava dor facial; a neuralgia foi considerada secundária a compressão vascular do bulbo. A teoria da compressão neurovascular na gênese da neuralgia vagoglossofaríngea é discutida de modo mais abrangente nos Comentários.

O diagnóstico diferencial inclui carcinoma, epitelioma e outras neoplasias da orofaringe e base do crânio, abscesso peritonsilar, cisto da glândula parótida, cisto nasofaríngeo, tumores do ângulo pontocerebelar, outras neuralgias (do n. V, do n. intermédio) e dor facial atípica. Nos abscessos peritonsilares e laringites a dor freqüentemente irradia-se para o ouvido 5. A distinção entre a neuralgia do n. intermédio e a variedade referida como neuralgia de Jacobson, na qual a dor é limitada ao ouvido e tuba auditiva, é particularmente difícil !0. O n. de Jacobson emerge do 
gânglio petroso e junta-se ao plexo timpánico no ouvido médio, juntamente com fibras dos nervos VII e X, nervos simpáticos e ramo auricular maior do plexo cervical, contribuindo na inervação do revestimento mucoso do tímpano, tuba auditiva e células da mastóide. A associação com a neuralgia trigeminal é conhecida, podendo tornar confuso o diagnóstico. Curiosamente, nesses casos, é sempre acometida a terceira divisão do n. V.

O tratamento com carbamazepina (na dose de $200 \mathrm{mg}$ três ou quatro vezes ao dia) pode trazer alívio completo da dor em alguns casos, mas os efeitos não são duradouros em geral.

Quanto à cirurgia, são de interesse histórico a exérese do processo estilóide através da fossa tonsilar, a neurectomia ou alcoolização do $\mathrm{n}$. laríngeo superior (que tem anastomoses com ramos faríngeos do $\mathrm{n}$. IX) e a neurotomia extracraniana do n. IX, ramo faríngeo do $\mathrm{n}$. X e gânglio simpático cervical superior M8. A tractomia bulbar de Sjõqvist permite a secção combinada de fibras convergentes dos nervos VII, IX, $X$ e V3, o que traz vantagem significativa nos casos de difícil diagnóstico diferencial. Essa cirurgia é tida como segura e sem seqüelas, porém é pouco difundida. A rizotomia percutânea por radiofrequiência do n. IX e seu gânglio petroso ao nível do forame jugular 12,16 tem sua maior indicação para o controle da dor neoplásica, trazendo como seqüela paralisia das cordas vocais e dificuldade de deglutição em graus variados. Pode ser indicada nos casos de neuralgia vagoglossofaríngea quando o paciente se encontra muito debilitado, tornando inaceitável o risco da abordagem direta da fossa craniana posterior.

A cirurgia de escolha é a secção intracraniana do n. IX proximal ao seu gânglio superior e dos filetes radiculares superiores do $n$. X, homolaterais à dor, através de craniectomia suboccipital paramediana ${ }^{4} »$ !8. A rizotomia intracraniana do n. IX para essa doença foi realizada com sucesso pela primeira vez por Dandy ${ }^{4}$; suas observações técnicas permanecem válidas mesmo com as vantagens proporcionadas pelo microscópio operatório e o aprimoramento da neuro-anestesia. A manipulação dos nervos pode ocasionar bradicardia e oscilações hemodinâmicas, devendo para isso atentar o cirurgião e o anestesista. A secção do n. IX pode causar taquicardia sinusal e hipertensão arterial transitórias; em alguns pacientes a compensação neurovegetativa pelo n. IX contralateral pode ser lenta, como no caso extremo relatado por Alonso e col. ${ }^{2}$, em que a pressão arterial permaneceu elevada por 4 meses. Pode ocorrer dificuldade transitória da deglutição, pela paralisia do $\mathrm{m}$. estilofaríngeo. Os reflexos faríngeo e do véu palatino são abolidos no lado denervado. A perda da gustação no terço posterior da língua em geral não é notada pelo paciente. A área de perda sensitiva não representa problema, estendendo-se pela porção posterior da língua, face, faringe superior até a epiglote, e palato mole. As fibras sensitivas do n. IX exercem um efeito inibitório sobre o mecanismo respiratório durante a deglutição, mas é questionável que uma rizotomia unilateral possa prejudicar essa função.

\section{CASUÍSTICA}

Caso 1 - ESS, paciente do sexo feminino, com 49 anos de idade, registro HVC 104-68. Há 8 anos com paroxismos dolorosos de forte intensidade na faringe, à direita (D), com irradiação para as regiões profundas da hemiíace e mandíbula homolaterais, acompanhados de hipoacusia subjetiva e tinnitus à D. Exames pré-operatórios não disponíveis para revisão. Cirurgia: secção intracraniana do n. IX direito, exclusivamente, com alívio imediato da dor Assintomática no seguimento' por 10 anos.

Caso 2 - PGC, paciente do sexo feminino, com 44 anos de idade, registro HVC 697-79. Há 8 anos com paroxismos dolorosos, em surtos extremamente fortes com periodos de remissão, início na faringe à $D$ e irradiação para o ouvido e ramo da mandíbula do mesmo lado, acompanhados de intensa salivação. Estímulos desencadeantes: deglutição, fala e inspiração profunda. Exames pré-operatórios: radiografias do crânio, planigrafias da base do crânio e articulações temporomandibulares e angiografia vertebrobasilar consideradas normais. Cirurgia: secção intracraniana do $n$. IX e dois filetes radiculares do $n$. $X$, à D. Alívio da dor, com dificuldade de deglutição e rouquidão transitórias.' Assintomática no seguimento por dois meses.

Caso 3 - HT, paciente do sexo feminino, com 74 anos de idade, registro HVC $330-89$. Há 18 meses com paroxismos dolorosos na garganta e base da língua, à D, irradiados para o ouvido (onde a dor torna-se mais intensa), precipitados pela fala e deglutição. Tomografia 
computadorizada (CT) do encéfalo e base do crânio normal. Cirurgia: secção intracraniana do n. IX e radicelas superiores do $n$. $X$, à $D$; observada a. basilar alongada e tortuosa, comprimindo o bulbo ao nível da zon»a de entrada dos nervos iX-X. Alívio transitório da dor, que retornou após algumas semanas em paroxismos restritos a base da língua, à direita. Novos exames: CT do encéfalo e angiografia vertebrobasilar, que demonstraram apenas sinais da craniectomia pregressa, alongamento e tortuosidade da a. basilar e sinais de aterosclerose. Reoperada após 10 meses, sendo a a. basilar envolta em tela de teflon e afastada da zona de entrada dos nervos IX-X. O alívio da dor persiste 4 meses após a segunda cirurgia.

\section{COMENTÁRIOS}

1. Sobre algumas implicações clínicas da inervação sensitiva das regiões profundas da face e transição cervicofacial (Fig. 1)

Define-se uma neuralgia pela dor no território de um nervo ou grupo de nervos. A inervação nociceptiva 5,15 da face, orofaringe, ouvido e transição cervicofacial é provida por fibras dos nervos cranianos V, VII, IX, X e XI, nervos espinhais C1, C2 e C3 e nervos simpáticos, que se anastomosam livremente a nível periférico e convergem no núcleo do tracto espinhal do n. V15. Descrevemos a seguir as principais anastomoses do n. IX e X, segundo Lazorthes ${ }^{11}$ : o n. IX comunica-se com o n. V (nos terminais linguais), n. VII (pelos nervos petrosos e alça de Haller), n. X (por filetes nervosos entre os gânglios petroso e jugular e no plexo faríngeo) e com $\mathrm{a}^{\wedge}$ cadeia simpática cervical (anastomoses diretas na base do crânio; ramos carótico-timpânicos); o n. X anastomosa-se com o n. VII (ramo auricular), n. IX, n. XI (cujo ramo interno acompanha o n. X) e com o n. XII (pelo gânglio nodoso), além de 2 ou 3 filetes nervosos entre o gânglio nodoso e o gânglio simpático cervical superior. A sensi-

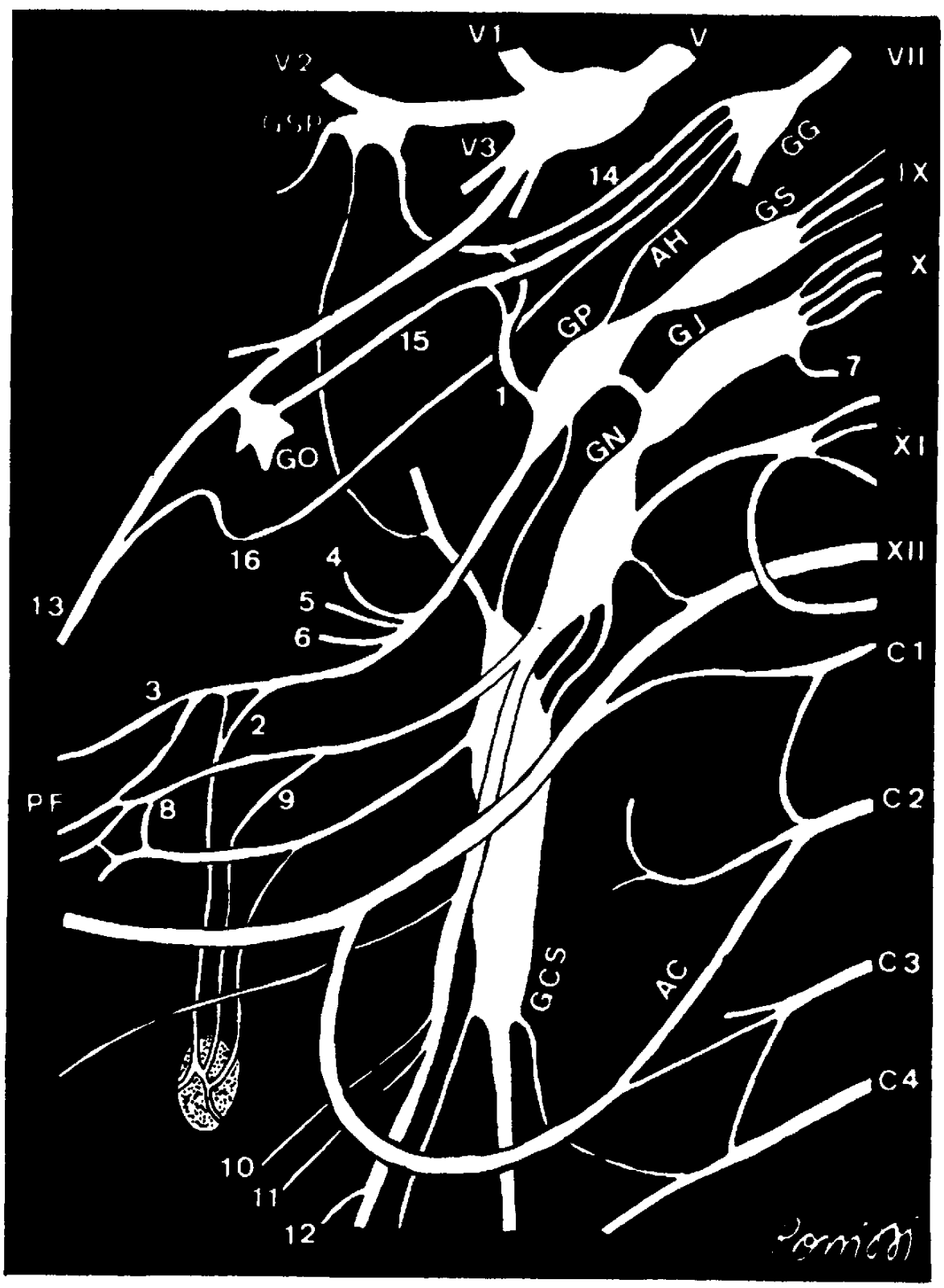

Fig. 1 - Ramos e anastomoses dos nervos cranianos IX $\boldsymbol{e} \boldsymbol{X}$.

$V(V 1,2,3), V I I, I X, X$, $X I, X I I=$ nervos cranianos; C 1, 2, 3, $4=$ nervos espinhais; GS, gânglio (ggio.) superior do n. IX; GP, ggio. petroso; GJ, ggio. jugular do $n$. $X ; G N$, ggio. nodoso ou plexiforme: GSP, ggio. esfenopalatino; GO, ggio. otico; GCS, ggio. cervical superior $e$ cadeia simpática; $A C$, alca cervical; $A H$, alca de Haller (inconstante); $P F$, plexo faringeo; $1, n$. de Jacobson ou timpanico; $2, n n$. carotideos do $n$. IX, incluindo n. de Hering; 3 , nn. faringeos do $n . I X ; 4, n$. do estilofaringeo; 5 , ramos tonsilares; 6 , n. do estiloglosso; 7 , ramo meníngeo do $n$. $X ; 8, n n$. faringeas do $n . X ; 9, n$. intercarotídeo; 10 , n. laringeo superior; 11, n. cardíaco superior; 12, n. laringeo recorrente; 13 , n. lingual; 14, n. petroso superficial maior; 15 , $n$. petroso superficial menor; 16, corda do timpano. 
bilidade dolorosa é, em condições normais, pouco desenvolvida e mal localizada na tonsila, faringe e base da língua e, menos ainda, na laringe 5; as sensações têm aqui um papel preponderante na integração reflexa. Nesse verdadeiro labirinto de vias nervosas é de se esperar que ocorram fenômenos dolorosos mal definidos, como as chamadas neuralgia esfenopalatina (de Sluder), carotidínea e neuralgia vidiana, entre outros, reunidos no grupo das algias faciais atípicas. Alguns relatos mais recentes demonstram que, na região cervicofacial, manifestações dolorosas muito semelhantes podem dever-se a vias anatômicas muito diferentes.

Observamos paroxismos de dor faríngea em alguns pacientes durante punção suboccipital lateral (no espaço atlanto-occipital), realizada para coleta de líquido cafalorraqueano ou mielografia cervical ${ }^{15}$. A dor é aguda, permanecendo em menor intensidade pelo tempo em que a agulha permanece no local onde a desencadeou e cessando com sua retirada; a descriçãa pelos pacientes assemelha-se a um tique doloroso, porém não ocorrem irradiação aural e outros fenômenos associados. Nenhum paciente era portador de neuralgia vagoglossofaríngea e, no seguimento, não se observou qualquer outro sintoma irritativo local. Atribuimos o fato a irritação do primeiro nervo cervical $(\mathrm{Cl})$, que se anastomosa com os nervos cranianos inferiores e com a cadeia simpática; outra possibilidade, que não descartamos, é considerar esse fato no contexto da dor referida, haja vista a densa convergência anatômica sensitiva no núcleo do tracto espinhal do n. V.

Um fenômeno até certo ponto semelhante foi denominado síndrome glossocervical («neck-tongue syndrome») por Lance e Anthony 8, que relataram 4 pacientes com queixa de dor unilateral na nuca ou região occipital, com ou sem sensação de dormência sobre essas áreas, e dormência na língua do mesmo lado, que se precipita pela rotação lateral brusca da cabeça (para qualquer lado, porém com sintomas mais intensos à rotação contralateral). Esses autores encontraram na literatura apenas dois casos semelhantes, descritos por Cyriax em 1962, em um dos quais a parestesia estendia-se até a porção posterior do véu palatino. A explicação aventada foi uma subluxação anormal da articulação atlanto-axial lateral e compressão do ramo ventral do n. C2 contra os processos articulares subluxados. Estudo da anatomia dinâmica desse mecanismo foi realizado por Bogduk 3. A conexão anatômica entre o n. C2 e o n. lingual dá-se pelo n. XII, fornecendo explicação plausível para a dor occipitonucal e parestesias linguais. Devemos notar que essa mesma via anatômica conecta-se também ao n. $\mathrm{Cl}$, que pode ser comprimido de modo mais evidente em sua passagem entre o atlas e a a. vertebral, durante a rotação da cabeça (a rotação extrema pode provocar estreitamento tão importante desse espaço que causa, em algumas pessoas, sintomas de insuficiência vértebro-basilar pela diminuição do fluxo sangüíneo pela a. vertebral).

Estímulos gustatórios podem desencadear tiques dolorosos vagoglossofaríngeos e a salivação profusa acompanha alguns casos em virtude do desequilíbrio autonômico associado ao paroxismo. Sintomas semelhantes foram observados em pacientes com pan-disautonomia aguda e, mais recentemente, em um caso de lesão das fibras simpáticas peri-carotídeas. $\operatorname{Truax}^{\wedge}$ relatou a ocorrência de dor paroxística na região da parótida e hiperemia pré-auricular, precipitadas por estímulos gustatórios na ausência de obstrução mecânica do dueto de Stenson (todos os estímulos desencadeantes eram aqueles normalmente relacionados a salivação, em especial os sabores doce e ácido); a dor era evanescente, desaparecendo após 10 a 30 segundos; os paroxismos iniciaram-se algumas horas após a realização de endarterectomia carotídea cervical homolateral, na qual o n. XII foi também inadvertidamente lesado. Foram documentadas vasodilatação parotídea assimétrica e disfunção simpática na pele sobre a parótida, sugerindo que os sintomas foram devidos a interrupção das fibras vasoconstrictoras simpáticas para essa glândula, permitindo intensa vasodilatação aos estímulos gustatórios, mediada pela atividade parassimpática desenfreada. Discutiu-se ainda o possível envolvimento do polipeptídeo intestinal vasoativo (VIP) na gênese dos sintomas. O curso dessa disfunção foi auto-limitado, com abrandamento progressivo dos sintomas em um ano.

Deve ainda ser mencionada a dor referida hemifacial, predominantemente aural, decorrente da compressão ou infiltração neoplásica do $\mathrm{n}$. X em sua passagem junto a traqueia, brônquio principal e linfonodos mediastinais, o que ocorre mais freqüentemente com o n. X direito, ou ainda da irritação do n. X relacionada a hérnia hiatal. Como já mencionamos, vias anatômicas e entidades nosológicas distintas podem oca- 
sionar manifestações dolorosas muito semelhantes na região cervicofacial. Deve-se proceder com muita cautela no diagnóstico diferencial.

\section{Sobre a teoria da compressão neurovascular}

A compressão pulsátil e distorção dos filamentos radiculares dos nervos cranianos por uma artéria alongada (decorrente do processo de envelhecimento) ou mesmo por uma veia, ao nível da área juncional entre a mielina central e periférica, foi apontada como causa de disfunções hiperativas desses nervos, como a neuralgia trigeminal (n. V), espasmo hemifacial (n. VII), tinnitus e vertigem (n. VIII), hipertensão arterial essencial (n. X esquerdo), além da neuralgia vagoglossofaríngea (n. IX-X). Algumas rizopatias cranianas «hipoativas» seriam também devidas a compressão neurovascular: neuropatía trigeminal, paralisia de Bell, neuronite vestibular e neuropatía hipoglossal 6a $\mathbf{a}_{\|}$assunto é controverso, e encontra-se minuciosamente discutido nos artigos de JannettaQ e de Adams i. Com base nesse modelo teórico, a descompressão microvascular dos nervos envolvidos tem alcançado bons resultados em cerca de dois terços dos pacientes operados, mas é questionável se esses resultados são devidos à «descompressão» do nervo 6 ou se a dissecção e manipulação dele produzem um trauma «menor» porém suficiente para interferir em sua atividade irritativa anormal i. Notem-se alguns problemas dessa teoria: a presença de contatos neurovasculares em pessoas assintomáticas e a ausência deles em pacientes sintomáticos; a presença de contatos similares em nervos que não são susceptíveis de disfunções hiperativas (e.g. n. XII e a. vertebral); os períodos de remissão; sendo as alterações vasculares referidas decorrentes do avanço da idade, seria de se esperar que a prevalência dessas disfunções aumentasse proporcionalmente a idade dos pacientes, o que não ocorre. Sumariamos a seguir algumas notas de anatomia e problemas específicos dessa teoria na gênese da neuralgia vagoglossofaríngea. Segundo as observações anatômicas de Lang e Reiter', o n. IX mede em sua porção intracisternal 15,65 (10-20)mm, sendo mais longo à esquerda $(15,95 \mathrm{~mm})$ e nos homens $(16,03 \mathrm{~mm})$ do que à direita $(15,26 \mathrm{~mm})$ e nas mulheres $(14,86 \mathrm{~mm})$; sua origem aparente na área retro-olivar do bulbo situa-se $3,19 \quad(1,5-5,0) \mathrm{mm}$ lateral à oliva inferior, e $1,79(1,0-3,0) \mathrm{mm}$ caudal ao sulco bulbopontino, emergindo segundo Lazorthes $\mathrm{n}$ em 4 ou 5 radicelas; sua porção central (glial) mede cerca de $0,1 \mathrm{~mm}$ nas radicelas mais mediais (motoras) e $1,1 \mathrm{~mm}$ nas laterais (sensitivas). $\mathrm{O}$ n. $\mathrm{X}$ tem 8,65 (4-15) radicelas, que emergem imediatamente abaixo daquelas do n. IX, numa extensão de $2,7(1,5-4,0) \mathrm{mm}$ rostral a $3,62(2,0-4,0) \mathrm{mm}$ caudal à oliva inferior, e apresentam-se em sua porção intracisterrtal divididas em dois grupos: o grupo superior mede 15,33 $(10,5-21) \mathrm{mm}$ de extensão $(14,72 \mathrm{~mm}$ à direita e $15,78 \mathrm{~mm}$ à esquerda, e $15,64 \mathrm{~mm}$ nos homens e $14,75 \mathrm{~mm}$ nas mulheres) e o grupo inferior $15,6(11,0-20,0) \mathrm{mm}$ de extensão $(15,07 \mathrm{~mm}$ à direita e $15,99 \mathrm{~mm}$ à esquerda, e $16,0 \mathrm{~mm}$ nos homens e $14,89 \mathrm{~mm}$ nas mulheres); sua porção central mede $0,1 \mathrm{~mm}$ nas radicelas mais mediais e até $2 \mathrm{~mm}$ nas laterais. Citamos apenas as mensurações estatisticamente significantes. Contatos neurovasculares são possíveis entre a zona juncional central-periférica desses nervos e a a. cerebelar posterior inferior (mais freqüente), a. vertebral, a. basilar, a. cerebelar anterior inferior e veias, isoladamente ou associados. Em uma série pessoal de 11 pacientes operados, Jannetta 6 realizou descompressão do n. IX-X em 9 casos (obtendo alívio duradouro em 6) e não encontrou vasos anormais em 2 casos (nos quais realizou a rizotomia clássica). Kempe e Smith 7 relataram um caso de a. hipoglossal persistente associada a neuralgia «glossofaríngea» (sic) e outras compressões neurovasculares sintomáticas por anastomoses carótico-basilares persistentes: a. trigeminal primitiva associada a neuralgia trigeminal (V2 e V3), artéria acústica primitiva associada a espasmo hemifacial e neuralgia do $\mathrm{n}$. intermédio (uma combinação muito rara). Note-se que o segmento central dos nervos IX-X é nas radicelas sensitivas (laterais) da ordem de 1 a 2 milímetros; compressões vasculares que corroboram a teoria de Jannetta devem dar-se precisamente nesse local e não no restante do curso intracisternal dos nervos (com extensão da ordem de 1,5 centímetros). Já mencionamos alguns problemas gerais dessa teoria. No caço específico da neuralgia vagoglossofaríngea, a não ocorrência de espasmos do m. estilofaríngeo e espasmos hemifaríngeos, por presumida irritação do n. IX e n. X, respectivamente, depõe contra essa teoria.

\section{Conclusão}

A questão da etiologia da neuralgia vagoglossofaríngea permanece sem solução; na maioria dos casos não se observaram alterações anátomo-patológicas evidentes. O engano fundamental, em particular no caso da teoria da compressão neurovascular, 
talvez esteja no fato de o médico em geral ter dificuldade em imaginar a dor e o sofrimento na ausência de uma lesão «real», anatomicamente verificável. Esse problema conceituai já fora exposto há 6 décadas por Leriche 13, ao analisar o aparente paradoxo de «doenças sem lesão» e da «doença anatômica sem expressão clínica»; na construção da patologia, Leriche insistia em seu caráter global, considerando a lesão «fruto possível de uma perversão funcional que precede o estado anatômico» ${ }^{13}$.

talvez esteja no fato de o médico em geral ter dificuldade em imaginar a dor e o sofrimento na ausência de uma lesão «real», anatomicamente verificável. Esse problema conceituai já fora exposto há 6 décadas por Leriche 13, ao analisar o aparente paradoxo de «doenças sem lesão» e da «doença anatómica sem expressão clínica»; na construção da patologia, Leriche insistia em seu caráter global, considerando a lesão «fruto possível de uma perversão funcional que precede o estado anatómico» ${ }^{13}$. REFERÊNCIAS

1. Adams CBT. Microvascular compression: an alternative view and hypothesis. J Neurosurg 1989, 70:1.

2. Alonso T, Pujol J, Ayuso A, Pujol J. Sustained hypertension after section of the glossopharyngeal nerve. J Neurol Neurosurg Psychiat 1981, 44 : 369

3. Bogduk N. An (anatomical basis for the neck-tongue syndrome. J Neurol Neurosurg Psychiat 1981, 44:202.

4. Dandy WE. Glossopharyngeal neuralgia (tic douloureux): its diagnosis and treatment. Arch Surg 1927, $15: 198$

5. Freystadtl B. Kehlkopf und Rachen in ihren Beziehungen zu den Erkrankungen des Zentralnervensystems. Berlin: Karger, 1928, p 45.

6. Jannetta PJ. Neurovascular compression in cranial nerve and systemic disease. Ann Surg 1980, 192-518

6 Janetta PJ Cranial rhizopathies. In Youmans JR (ed): Neurological Surgery, vol 6 Ed 3. Philadelphia: Saunders, 1990, p 4169.

7. Kempe LG, Smith DR. Trigeminal neuralgia, facial spasm, intermedius and glossopharyngeal neuralgia with persistent carotid-basilar anastomosis. J Neurosurg 1969, 31:445.

8. Lance JW, Anthony M. Neck-tongue syndrome on sudden turning of the head. J Neurol Neurosurg Psychiat 1980, 43 : 97.

9. Lang J, Reiter U. Ueber die intrazisternale Länge der Hirnnerven VII-XII. Neuro-ᄀ chirurgia (Stuttgart) 1985, 28:153.

10. Laskiewicz A. Anatomical and clinical considerations on some rare forms of glossopharyngeal neuralgias. Acta Oto-Laryngol 1953, 43 : 545.

11. Lazorthes G. Le Systeme Nerveux Périphérique. Paris: Masson, 1955, p 127 (n. IX), p 139 (n. X).

12. Lazorthes Y, Verdie JC. Radiofrequency coagulation of the petrous ganglion in glossopharyngeal neuralgia. Neurosurgery 1979, 4:512.

13. Leriche R. La Philosophie de la Chirurgie. Paris: Flammarion, 1951, p 16.

14. Mracek Z. Compression of the spinal trigeminal tract due to pressure of the inferior posterior cerebellar artery as the causative factor of facial neuralgia (Cze). Rozhledy $v$ chirurgii 1982, $61: 116$.

15. Rossitti SL, Araújo JFM, Zuiani AR, Balbo RJ. Dor faríngea durante punção suboccipital lateral. Arq Neuro-Psiquiat (São Paulo) 1989, 47:182.

16. Tew JM Jr. Percutaneous rhizotomy in the treatment of intractable facial pain (trigeminal, glossopharyngeal and vagal nerves). In Schmidek HH, Sweet WH (eds): Current Techniques in Operative Neurosurgery. New York: Grune \& Stratton, 1977, p 409.

17. Truax BT. Gustatory pain: a complication of carotid endarterectomy.' Neurology 1989, 39:1258.

18. White JC, Sweet WH. Pain and the Neurosurgeon: A Forty-Year Experience. Springfield: Charles C. Thomas, 1969, p 257. 